\title{
Genetic Disorders of Insulin Action: Far More than Diabetes
}

\author{
Benjamin G. Challis • Robert K. Semple
}

Published online: 13 September 2013

(C) Springer Science+Business Media New York 2013

\begin{abstract}
As obesity rates inexorably rise, so the incidence of diseases associated with insulin resistance (IR) is also surging. The tendency towards obesity-associated IR appears significantly genetically determined, but the underlying genetic determinants have proved difficult to elucidate. In contrast, genetic study of unusually severe IR, often presenting in childhood and/or in lean patients, has uncovered many examples of causative single gene defects. "IR" is usually defined by reduced ability of insulin to lower blood glucose, but study of such patients suggests the existence of IR subphenotypes, and implies that some harmful components of the "IR syndrome" actually require intact insulin action in some pathways or tissues. Recently several genetic disorders have also been identified that are due to mutations activating insulin signaling to produce hypoglycemia or overgrowth. This article will discuss these groups of disorders, emphasizing their clinical recognition, and the implications of genetic diagnosis for understanding of common disease.
\end{abstract}

Keywords Insulin resistance $\cdot$ Diabetes $\cdot$ Hypoglycemia Genetic disorders · Insulin receptor · AKT2 · PI3 kinase · Lipodystrophy · Growth · Fatty liver · PCOS · Segmental overgrowth $\cdot$ Insulin action

B. G. Challis $\cdot$ R. K. Semple $(\bowtie)$

Wellcome Trust-MRC Institute of Metabolic Science, The University of Cambridge Metabolic Research Laboratories, Cambridge CB2 0QQ, UK

e-mail: rks16@cam.ac.uk

B. G. Challis

e-mail: bc340@medschl.cam.ac.uk

\section{Introduction}

Insulin, the quintessential "hormone of plenty", is required for survival and without it severe catabolism, cachexia and death ensue despite adequate caloric intake. This is clearly attested to by the many harrowing accounts of the natural history of type 1 diabetes in the pre-insulin era, and by the transformational effects of exogenous insulin [1]. This has turned type 1 diabetes from a death sentence into a chronic disease where most clinical research effort now focuses on small incremental improvements in long term risk factor reduction.

Although complete insulin deficiency is clinically devastating, it is impaired insulin action which has emerged as a major medical battleground in the early twenty first century by virtue of the soaring prevalence of obesity-related "insulin resistance", and the wide range of pandemic diseases - including atherosclerosis [2], fatty liver disease [3], ovulatory dysfunction [4] and cancer [5] as well as type 2 diabetes [6] - with which this is associated. Yet the gestation of insulin resistance as a concept has been slow and difficult, despite the seminal observations of Harold Himsworth as early as the 1930s that some people with diabetes showed a blunted hypoglycemic effect of exogenous insulin [7] and understanding of its underlying basis remains fragmentary.

Since sequencing of the gene encoding the insulin receptor in 1985 [8] determined work from many laboratories has uncovered an elaborate signaling network transducing insulin binding into important metabolic and growth effects on target cells and tissues. In parallel, genetic studies of rare individuals with severe disorders of insulin action have identified increasing numbers of single gene defects that produce either extreme IR, or, more recently, insulin-independent hypoglycemia [9••] or tissue growth $\left[10^{\bullet}\right]$. This review will focus on clinical 
identification of patients in whom genetic testing for a disorder of insulin action is warranted, will summarize key aspects of their management, and will discuss the lessons for common disease. To prime this, current understanding of cellular insulin action will briefly be outlined.

\section{Overview of the Insulin Signaling Network}

The cellular and molecular mechanisms of insulin signaling have been extensively reviewed elsewhere [11], thus only a brief overview will be presented here, as summarized in Fig. 1.

The actions of insulin are mediated by a single, dimeric transmembrane receptor protein. Insulin binding to the extracellular subunits of its receptor induces activation of tyrosine kinase activity of the intracellular subunits, which then phosphorylate each other and subsequently several downstream effector molecules. These include insulin receptor substrate (IRS) proteins, of which IRS1 and IRS2 are most important for insulin's metabolic and growth effects. Phosphorylated IRS-1 or IRS-2 recruit and activate proteins, including phosphatidylinositol 3kinase (PI3K), which binds via a $\mathrm{p} 85$ regulatory subunit, and the growth factor receptor binding protein 2 (GRB2). PI3K adds a phosphate group to the membrane phospholipid phosphatidylinositol bisphosphate $\left(\mathrm{PIP}_{2}\right)$ to produce phosphatidylinositol 3,4,5-triphosphate $\left(\mathrm{PIP}_{3}\right)$. It is the appearance of $\mathrm{PIP}_{3}$ in the plasma membrane that recruits and activates the three subtypes of the protein kinase AKT, also known as protein kinase $\mathrm{B}$, and it is these kinases that directly target proteins that activate cellular growth or alter metabolism. For example, direct substrates of AKT are involved in repressing apoptosis, stimulating glucose uptake by the glucose transporter GLUT4, driving new synthesis of fatty acids, increasing glycogen accumulation, and repressing expression of gluconeogenic enzymes in the liver and lipolysis of triglyceride in adipose tissue, among many other effects [12]. GRB2, in contrast, stimulates the MAP kinase/ extracellular signal-regulated kinase (MAPK/ERK) pathway that traditionally is viewed as stimulating cell growth and gene expression.

\section{Genetic Forms of Severe Insulin Resistance}

What is Insulin Resistance?

Despite insulin's complex and widespread effects on tissue metabolism and growth, IR is generally defined solely as a diminution in the ability of a given concentration of insulin to lower plasma glucose levels. However it has long been speculated that defects in insulin action that are selective to one or more of its actions may exist [13]. As will be discussed, clinical

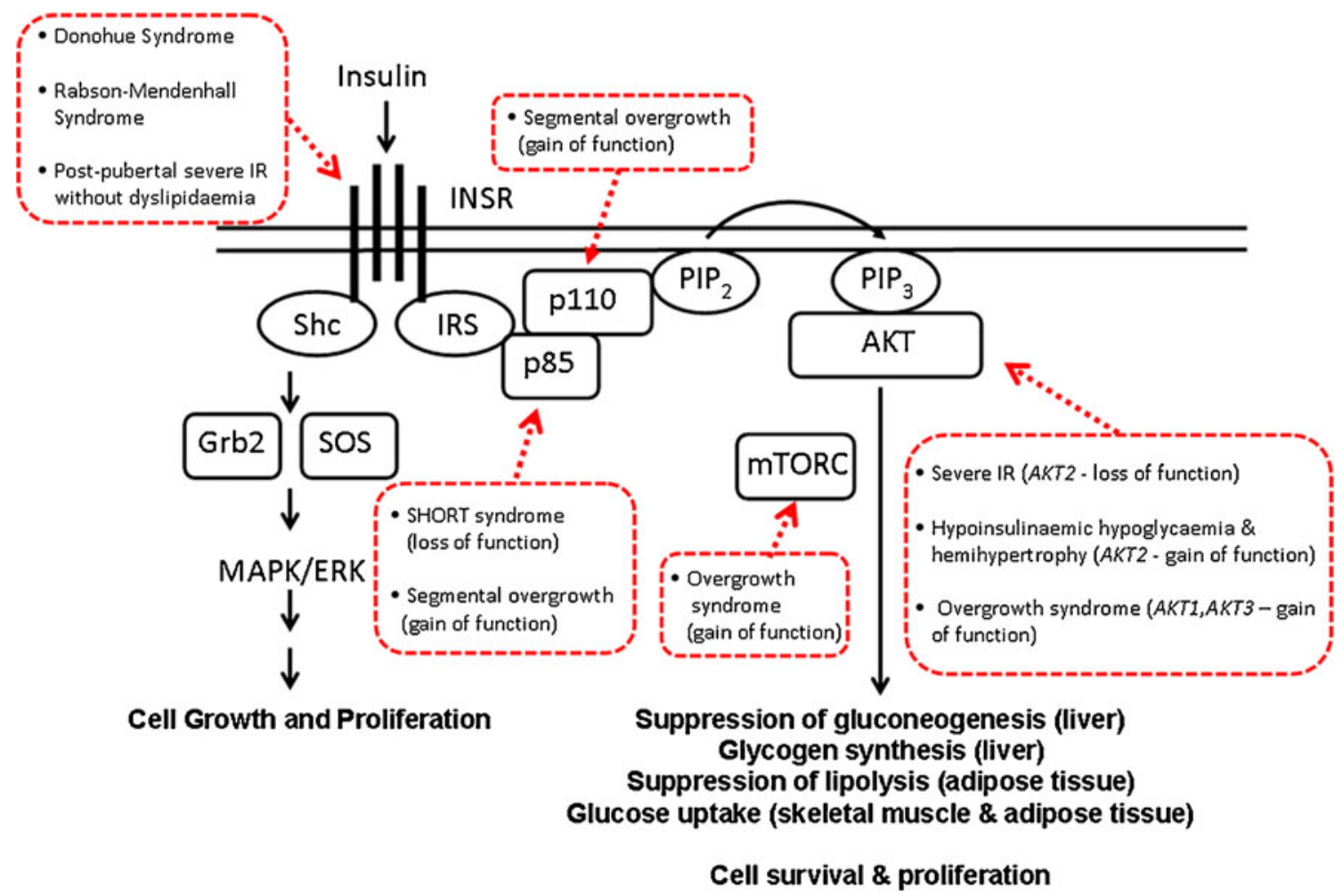

Fig. 1 1Simplified schematic of key insulin signaling pathways, including the MAPK/ERK and PI3K-AKT pathways. Sites of known pathogenic mutations and their corresponding syndromes are shown by the dashed red arrows and boxes, respectively 
support for this idea has now begun to emerge from human genetic disorders of insulin action.

Because there is a continuum of sensitivity to insulin, setting thresholds for the diagnosis of "severe" IR is arbitrary. Nevertheless, in patients with complete endogenous insulin deficiency, an exogenous insulin requirement of $3 \mathrm{U} / \mathrm{kg} /$ day is a commonly used cut-off [14॰•]. Alternatively, in a patient without diabetes or impaired glucose tolerance a fasting plasma insulin $>150 \mathrm{pmol} / \mathrm{l}$ or a disproportionately high peak insulin on an oral glucose tolerance test of $>1500 \mathrm{pmol} / 1$ would be suggestive of a syndrome of severe IR. A key factor to consider, however, is the degree of adiposity. These thresholds are useful if applied to those without obesity, but are insufficiently specific in obese patients, as more prevalent forms of IR that are driven by obesity come to dominate in this setting, and long experience suggests that the chances of finding a single gene defect explaining IR are consequently much lower. Moreover many patients presenting for clinical evaluation have partial beta cell decompensation making insulin levels difficult to interpret due to hyperglycemia. For these reasons the clinical history and examination, and ancillary biochemical findings are extremely important in selecting patients for further investigation for possible genetic severe IR.

\section{Generic Clinical Features of Severe Insulin Resistance}

Most patients with severe IR are clinically identifiable long before they have symptomatic diabetes, and in some several years of postprandial hypoglycemia, which may be severe, can be seen. However nearly all manifest one or more clinical features apart from perturbed glucose homeostasis that are generic to all forms of genetic severe IR. Acanthosis nigricans (AN) is among the most reliable clinical signs. It is characterized by a velvety, hyperpigmented and hyperkeratotic appearance of skin in the axillae, nuchal region, groin and other flexural areas, and is commonly associated with skin tags. AN gradually fades as beta cells decompensate, and may not be seen in with poorly controlled diabetes. However, patients or their parents may give a clear history of their having had a "dirty" neck from early childhood. AN is also seen in obesityrelated IR, and rarely may be familial without IR or may be seen as a paraneoplastic phenomenon [15].

The commonest clinical presentation of a female with severe IR is with the cosmetically distressing clinical hyperandrogenism, usually with oligomenorrhea or amenorrhoea. Investigation characteristically reveals biochemical hyperandrogenism of ovarian origin and the sonographic appearance of polycystic ovaries. Biochemical hyperandrogenism may be severe, with testosterone levels reaching the low male range, but is usually reversed with GnRH suppression. In our practice we have seen several patients who have developed small virilizing tumors in the context of many years of benign severe
IR-related hyperandrogenism [16], so even when severe IR is diagnosed caution is warranted.

Other features of severe IR include abnormal adipose tissue amount or distribution, or a range of growth disorders, but these are seen in only some syndromes and so are considered separately.

\section{Genetic Defects in Insulin Signaling Molecules}

\section{Insulin Receptor}

Genetic defects in the insulin receptor itself may be divided into recessive conditions presenting in infancy or early childhood, and a milder disorder commonly presenting peripubertally in girls. Infantile recessive forms of insulin receptoropathy are commonly called Donohue syndrome (formerly leprechaunism) or Rabson Mendenhall syndrome, though these terms are rather variably applied. Donohue syndrome is most severe, being characterized by linear growth retardation with soft tissue overgrowth, producing a coarse facial appearance, severe AN and hypertrichosis, overgrowth of sex hormone-dependent tissues, and organomegaly, often with nephrocalcinosis. The core metabolic derangement consists of fasting hypoglycemia with postprandial hyperglycemia, very severely elevated plasma insulin, but no ketoacidosis even in the absence of insulin receptor function, at least for the first year or two of life.

Death most commonly occurs in Donohue syndrome in the first or second year of life [17, 18], often during intercurrent infection. Continuous feeding often helps to reduce oscillations between hypo- and hyperglycemia, but there is no clear evidence for efficacy of oral insulin sensitizing agents, and even high dose insulin may be ineffective. Subcutaneous recombinant human IGF-1 is often used based on a collection of case reports and case series reporting medium to longer term efficacy $[19,20]$. However, the literature is extremely difficult to interpret given the large number of different insulin receptor mutations, each likely to have different degrees of residual function, and the lack of controlled studies.

Like Donohue syndrome, Rabson Mendenhall syndrome is caused by mutations in both alleles of the insulin receptor gene, and takes its eponym from clinical descriptions in the $1950 \mathrm{~s}$ [21]. Its clinical features differ from those of Donohue syndrome largely in degree only, and the dividing line between the two syndromes is vague. Affected children initially have postprandial hyperglycemia which progresses to poorly controlled diabetes due to $\beta$-cell decompensation within $10-15$ years [22]. When this happens vast quantities of exogenous insulin may be required and glycemic control often remains suboptimal, leaving patients highly susceptible to advanced microvascular diabetic complications and diabetic ketoacidosis [23]. Prognosis has historically been poor with therapeutic options limited to insulin sensitizing agents, of which the mainstay is metformin, 
with scattered reports of GLP1 agonists or thiazolidinediones only, usually in combination with very high doses of replacement insulin. Recombinant human leptin has also been reported to improve glucose levels [24], and, as for Donohue syndrome, rhIGF1 is frequently used, though once again, the evidence for its use is piecemeal only.

Milder insulin receptor defects presenting at or beyond puberty are much more common than Donohue or Rabson Mendenhall syndrome and are generally caused by mutations affecting the intracellular domain of the receptor. They are usually autosomal dominant, but examples of recessive inheritance have also been reported. The resulting syndrome is a combination of the generic features of IR described above in a severe form; by far the most common presentation is that of a lean girl between 10 and 16 years old with severe hyperandrogenism and oligomenorrhea or amenorrhoea, well advanced puberty and severe AN [25-27]. Symptoms suggestive of postprandial hypoglycemia may also be reported. Severe IR often remains undiagnosed in affected men for many decades, due to failure to understand the significance of AN in a lean man.

Hyperglycemia, AN and ovarian dysfunction are common to genetic insulin receptor defects and lipodystrophy. However the insulin receptoropathies diverge from these other groups in some respects: despite extreme IR, patients with insulin receptor defects do not exhibit elevated triglycerides, or suppressed HDL cholesterol, or fatty liver [23, 28]. Moreover, instead of showing the suppressed plasma levels of the liverderived proteins SHBG and IGFBP1 and the fat cell-derived protein adiponectin, well known in most forms of IR, they show preservation or elevation of each of these markers [29-33]. This has practical utility in discriminating patients with severe IR likely to have receptor defects, allowing targeted sequencing of the insulin receptor gene only in patients with a high pre-test probability of a mutation [32]. It also raises the possibility that some of the long term complications of prevalent IR, namely atherosclerosis and liver cirrhosis, may not be a feature of insulin receptoropathy, though this has yet to be assessed in long term studies. Finally, absence of these features from this prototypic, molecularly defined form of severe IR implies that they are linked not to loss of insulin action per se, but rather may be due to the harmful effects of hyperinsulinemia exerted on relatively preserved signaling pathways or tissues.

Current treatment for milder receptoropathies centers on dietary modification, early use of metformin at the maximum tolerated dose, and delivery of adequate doses of insulin, often using concentrated formulations [34], when secondary beta cell failure occurs. Use of other insulin sensitizers is rational, but evidence of efficacy is lacking. rhIGF-I may exert some beneficial effects, but evidence for long terms risks and benefits is not established [19, 20,22]. Oral contraceptive pills may be used to suppress gonadotrophins, sometimes with anti-androgens added in addition to treat hyperandrogenism. A more radical approach which has the potential to normalize androgens even in the face of the most severe IR-related elevation is the use of potent $\mathrm{GnRH}$ agonists with hormone replacement therapy - a form of "block and replace" therapy which we have found effective in a handful of patients.

\section{Postreceptor Defects}

With the identification of the molecules involved in transmitting the signal from the activated insulin receptor into cells, it was anticipated that many unexplained cases of severe early onset IR in lean patients would be due to genetic loss of function of these components. In general this has proved not to be the case, however the small number of convincing loss-offunction mutations in insulin signaling genes identified over the
Table 1 Classification, genetic basis and discriminating features of syndromes resulting from impaired or overactive insulin signalling
Impaired insulin signalling

A) Proximal signalling defects INSR

PIK3R1

B) Distal signalling defects

$A K T 2$, others to be defined

Overactivation of insulin signalling $A K T 2[9 \bullet \bullet]$

$\operatorname{PIK} 3 C A[10 \bullet \bullet, 35 \bullet, 36 \bullet \cdot]$, $\operatorname{MTOR}[35 \bullet \bullet]$

PIK3R2, AKT3[35••, 36••]

\section{Discriminating Features}

Usually lean

Extreme hyperinsulinaemia, normal lipid profile, normal or elevated adiponectin, SHBG and IGFBP1

\section{SHORT syndrome}

Insulin resistance, normal lipid profile, normal adiponectin

Dependent on specific signalling defect due to selective impairment on some intracellular arms of the insulin signalling pathway

Hypoketotic Hypoinsulinaemic Hypoglycaemia

Left Sided Hemihypertrophy

Segmental Overgrowth Syndrome

Megalencephaly-Polymicrogyria-Polydactyly-Hydrocephalus (MPPH), Megalencephaly-capillary malformation (MCAP) 
past decade (Table 1) have been very informative about the nature of prevalent IR, and have raised questions about its nature.

The first postreceptor defect to be reported was a heterozygous missense mutation in AKT2, which was found in four members of a single family [37]. Interestingly, and quite unlike patients with insulin receptor defects, affected patients had severe fatty liver and dyslipidemia, and the one female formally assessed had some deficiency of adipose tissue, or lipodystrophy, particularly affecting femorogluteal fat depots [28]. The striking metabolic differences between insulin receptor defects and this syndrome due to an AKT2 mutation could be interpreted either as supporting the existence of an insulin signaling pathway branching upstream from AKT2 that drives liver fat accumulation, and that is thus unprotected from the high levels of insulin needed to maintain euglycemia in the normal range, or as evidence that AKT2 plays a more important role in adipose tissue development or function than in hepatic response to insulin meaning that its deficiency leads to a predominantly lipodystrophic phenotype.

New evidence with the potential to throw further light on this issue has very recently been reported with the finding that SHORT syndrome (Short stature; Hyperextensibility of joints and/or Hernia (inguinal); Ocular depression (deep-set eyes); Rieger anomaly (defective development of the anterior chamber of the eye that can lead to glaucoma); Teething delay) is caused by dominant mutations affecting the p85 regulatory subunit of phosphatidylinositol 3 kinase (PI3K). PI3K, as discussed above, is a critical intermediate between the insulin receptor and AKT2 activation $[38 \bullet \bullet, 39 \bullet, 40 \bullet \cdot$. A high prevalence of IR was reported in the patients with PI3K mutations across the three recent reports, and many patents were also reported to exhibit lipodystrophy affecting subcutaneous adipose tissue in several depots including the face and femorogluteal regions. However, very interestingly, one of the three reports suggested that SHORT syndrome does not feature an abnormal lipid profile, or fatty liver, or suppressed adiponectin levels [40••].

Collectively these findings argue for the existence of a distinct biochemical syndrome produced by very proximal insulin signaling defects, namely severe IR with normal lipid triglycerides, HDL cholesterol and no fatty liver, and usually preserved or elevated levels of adiponectin. Finding this profile should trigger first genetic sequencing of the gene for the insulin receptor, and then enrolment into a research study to look at other proximal insulin signaling genes. The question of the mechanism explaining the very different phenotype of patients with AKT2 mutations, although at first sight rather recherché given their rarity, is potentially highly important, as the identification of a pathway explaining the high prevalence of fatty liver and dyslipidemia in IR that could potentially be targeted for therapeutic benefit in common disease would be of great value. No specific evidence has been published regarding clinical management of these rare patients with postreceptor insulin signaling defects, and so at present following the same principles established for insulin receptoropathy and, where relevant, lipodystrophy, is reasonable at present.

\section{Lipodystrophic Insulin Resistance}

Perhaps the major theme emerging from genetic research into unexplained forms of severe IR over the past 20 years has been the great importance of healthy adipose tissue for maintaining metabolic health. Thus, the rare recessive congenital generalized lipodystrophy, in which there is a nearly global absence of metabolic adipose depots from birth, is almost invariably associated with an exaggerated form of "prevalent" IR, with severe hypertriglyceridemia and fatty liver, and markedly suppressed HDL cholesterol seen in addition to generic features of IR. Of cases $95 \%$ are accounted for by mutations in either AGPAT2, which plays a key role in triglyceride synthesis [41, 42], or in BSCL2 [42], which encodes a more enigmatic protein which has been suggested to play roles in adipocyte differentiation and lipid droplet formation [43]. Some of the remaining cases have defects in the formation of small plasma membrane invaginations called caveolae, caused by mutations in either CAV1 [44] or PTRF [45]. In the case of PTRF mutations, myopathy is also seen, which is a key clinical pointer to the diagnosis.

More common than the generalized lipodystrophies are familial partial lipodystrophies (FPLD), in which adipose tissue is deficient in only some areas of the body. Just as for generalized lipodystrophies, these are commonly associated with severe fatty liver and dyslipidemia as well as severe IR. By far the most common genetic defects underlying FPLD occur in the genes LMNA or PPARG. LMNA encodes an intermediate filament component of the cytoskeleton around the nuclear envelope, and mutations in particular domains lead to loss of subcutaneous adipose tissue from the limbs and trunk, with preserved or increased adipose tissue intraabdominally and in the head and neck [46]. This can lead to a somewhat Cushingoid adipose topography, and to the erroneous impression of obesity in a dressed patient.

PPARG encodes a nuclear hormone receptor with properties well suited to function as a sensor of fatty acid flux, and which has shown to serve as the master regulator of fat cell differentiation [47]. Mutations causing autosomal dominant FPLD lead to a relative diminution of subcutaneous adipose tissue on the limbs, though this may sometimes be subtle, with severe dyslipidemia and often early onset hypertension [48]. A handful of rarer mutations have also been described, including defects in two different lipid droplet proteins, perilipin [49] and CIDEC [50], which play distinct roles in the mobilization of triglyceride stores from the single large lipid droplet in fat cells. Deficiency of CIDEC in a single patient has been shown to give rise to a unique multilocular appearance of 
residual white adipose tissue, quite unlike the usual single large lipid droplet normally seen [50].

Many questions remain to be answered about the cellular mechanisms linking these genetic defects to distinct distributions of adipose tissue deficiency. However viewed more generally, the striking shared feature among all these disorders is a paucity of femorogluteal subcutaneous adipose tissue, lending support to the concept that this adipose depot is particularly important for metabolic health. This is further supported by the relatively low prevalence of severe IR seen in acquired partial lipodystrophy, in which subcutaneous adipose tissue is usually lost in a craniocaudal sequence, arresting at the umbilicus [46]. On the other hand, as discussed above, several patients with SHORT syndrome and PI3K mutations have also been shown to exhibit deficiency of femorogluteal and other fat depots, and yet although they do show severe IR, they do not appear to exhibit dyslipidemia or fatty liver. This discordance has yet to be explained.

The central principle underlying management of lipodystrophy is the "offloading" of adipose tissue, based on its reduced or absent capacity to store excess dietary energy in the form of triglyceride. This may be achieved using a hypocaloric, low fat diet, but in cases with low leptin levels adjunctive subcutaneous leptin plays an important accessory role, probably by reducing the drive to eat, and thereby making dietary compliance easier [51-53].

\section{Complex Syndromes Featuring Severe Insulin Resistance}

Although largely beyond the scope of this review, it should be noted that severe IR may also be one component of several more complex genetic syndromes. In some there is severe IR which is unequivocally disproportionate to the degree of excess body fat. These include the progeria syndromes named after Werner and Bloom [54, 55], osteodysplastic primordial dwarfism of Majewski type 2 [56], a recently described syndrome featuring lipodystrophy, deafness and hypogonadism caused by a single amino acid deletion from the polymerase domain of DNA polymerase delta [57••. and Alström syndrome, caused by loss of the large centrosomal protein ALMS1 [58, 59]. Intriguingly, all of these syndromes feature mutations that disrupt mitosis and/or DNA damage repair, or interfere with centrosomal function. It is not clear at present why such a diverse range of defects should give rise to severe IR in the face of otherwise very different syndromes, and understanding any common underlying mechanism is likely to yield new insights into the maintenance of normal human insulin sensitivity.

\section{Genetic Activation of Postreceptor Insulin Signaling}

The genetics of human metabolic disease offers several examples of genes that have been shown to harbor either loss-offunction or gain of function having opposite effects on blood glucose to produce either diabetes or hyperinsulinemic hypoglycemia through effects on pancreatic beta-cell function. These include diabetes with loss-of-function mutations affecting the glucokinase gene [60] and hyperinsulinism caused by gain-of-function mutations [61], and, conversely, neonatal diabetes due to activating mutations affecting the hyperpolarizing AT-sensitive potassium channel, and hyperinsulinism caused by loss-of-function mutations [62]. However it is only very recently that any examples of mutations activating insulin signaling pathways have been described.

\section{Hypoketotic, Hypoinsulinemic Hypoglycemia}

We have recently reported a novel syndrome in three children of severe persistent hypoinsulinemic hypoglycemia associated with suppressed ketones and branched chain amino acids, preserved liver glycogen and left sided hemihypertrophy but with no detectable 'big' proIGF2. We demonstrated this to be due to a de novo mutation in AKT2 [9••] which effectively partly uncouples its activation from the need for upstream PI3K activity, and means that it is constantly mildly active even in the absence of insulin. This appears adequate to prevent physiological de-repression of hepatic gluconeogenesis in the postabsorptive and fasting states, and has meant that affected patients have required long-term percutaneous feeding to prevent nocturnal severe hypoglycemia.

\section{Segmental Overgrowth Syndromes}

Although it is tempting for those who study insulin signaling pathways in cells and tissues to regard molecules such as the PI3K complex and AKT as "insulin signaling molecules", it is important to remember that they form part of a highly conserved signaling pathway that is utilized downstream from many growth factor receptors, and indeed activating mutations in PI3K have been discovered in the past few years in a high proportion of cancers [63]. The importance of PI3K and AKT signaling in growth has recently further been emphasized by the findings that rare segmental overgrowth disorders, characterized by asymmetric and sometimes relentless excessive growth of only some parts of the body, are commonly accounted for by somatic activating mutations in PI3K or AKT $[10 \bullet \bullet, 35 \bullet, 36 \bullet \cdot$. Some of these disorders, notably those caused by PI3K activation, feature enormous increases in adipose tissue accumulation, yet no systemic metabolic disturbance has yet been reported despite the critical importance of PI3K for insulin-stimulated glucose uptake.

\section{Conclusions}

The problem of understanding how insulin action goes awry in common disease is a thorny one due to the many tissues 
involved in maintaining metabolic homeostasis, and due to the complex series of signaling responses elicited by insulin's actions on its nearly ubiquitous receptor. Genetic study of patients with severe perturbation of insulin action has already improved diagnosis and management of these rare conditions, and resourceful cellular and physiological studies of these "experiments of nature" promises to yield new insights in the coming years into the relationship between insulin's growth-promoting and metabolic effects in vivo, and into the nature of the "partial" IR that underlies the major pandemic pathologies associated with prevalent forms of IR.

\section{Compliance with Ethics Guidelines}

Conflict of Interest Benjamin G. Challis declare that he has no conflict of interest.

Robert K. Semple declare that he has no conflict of interest.

Human and Animal Rights and Informed Consent This article does not contain any studies with human or animal subjects performed by any of the authors.

\section{References}

Papers of particular interest, published recently, have been highlighted as:

•- Of major importance

1. Bliss M. Discovery of Insulin, Anniversary Edition edn. Chicago: University of Chicago Press; 2007.

2. Resnick HE, Jones $\mathrm{K}$, Ruotolo $\mathrm{G}$, et al. Insulin resistance, the metabolic syndrome, and risk of incident cardiovascular disease in nondiabetic american indians: the Strong Heart Study. Diabetes Care. 2003;26(3):861-7.

3. Preiss D, Sattar N. Non-alcoholic fatty liver disease: an overview of prevalence, diagnosis, pathogenesis and treatment considerations. Clin Sci (Lond). 2008;115(5):141-50.

4. Dunaif A, Segal KR, Futterweit W, Dobrjansky A. Profound peripheral insulin resistance, independent of obesity, in polycystic ovary syndrome. Diabetes. 1989;38(9):1165-74.

5. Gallagher EJ, LeRoith D. Minireview: IGF, insulin, and cancer. Endocrinology. 2011;152(7):2546-51.

6. Hanson RL, Imperatore G, Bennett PH, Knowler WC. Components of the "metabolic syndrome" and incidence of type 2 diabetes. Diabetes. 2002:51(10):3120-7.

7. Parker VE, Savage DB, O'Rahilly S, Semple RK. Mechanistic insights into insulin resistance in the genetic era. Diabet Med. 2011;28(12):1476-86.

8. Ullrich A, Bell JR, Chen EY, et al. Human insulin receptor and its relationship to the tyrosine kinase family of oncogenes. Nature. 1985;313(6005):756-61.

9. - Hussain K, Challis B, Rocha N, et al. An activating mutation of AKT2 and human hypoglycemia. Science. 2011;334(6055):474. First description of an activating mutation downstream of the insulin receptor that results in a syndrome of insulin-independent hypoglycaemia and hypertrophy.

10. • Lindhurst MJ, Parker VE, Payne F, et al. Mosaic overgrowth with fibroadipose hyperplasia is caused by somatic activating mutations in PIK3CA. Nat Genet. 2012;44(8):928-33. One of three publications demonstrating that somatic activating mutations in the PI3K-AktmTOR pathway result in segmental overgrowth.

11. Taniguchi CM, Emanuelli B, Kahn CR. Critical nodes in signalling pathways: insights into insulin action. Nat Rev Mol Cell Biol. 2006;7(2):85-96.

12. Schultze SM, Jensen J, Hemmings BA, et al. Promiscuous affairs of $\mathrm{PKB} / \mathrm{AKT}$ isoforms in metabolism. Arch Physiol Biochem. 2011;117(2):70-7.

13. Brown MS, Goldstein JL. Selective versus total insulin resistance: a pathogenic paradox. Cell Metab. 2008;7(2):95-6.

14. •- Semple RK, Savage DB, Cochran EK, et al. Genetic syndromes of severe insulin resistance. Endocr Rev. 2011;32(4):498-514. Comprehensive review describing the genetics of severe IR and presentation of a revised classification based on an improved understanding of the underlying pathophysiology of these genetic disorders.

15. Ellis DL, Kafka SP, Chow JC, et al. Melanoma, growth factors, acanthosis nigricans, the sign of Leser-Trelat, and multiple acrochordons. A possible role for alpha-transforming growth factor in cutaneous paraneoplastic syndromes. N Engl J Med. 1987;317(25):1582-7.

16. Brisigotti M, Fabbretti G, Pesce F, et al. Congenital bilateral juvenile granulosa cell tumor of the ovary in leprechaunism: a case report. Pediatr Pathol. 1993;13(5):549-58.

17. Donohue WL, Uchida I. Leprechaunism: a euphemism for a rare familial disorder. J Pediatr. 1954;45(5):505-19.

18. Kosztolanyi G. Leprechaunism/Donohue syndrome/insulin receptor gene mutations: a syndrome delineation story from clinicopathological description to molecular understanding. Eur J Pediatr. 1997;156(4):253-5.

19. Regan FM, Williams RM, McDonald A, et al. Treatment with recombinant human insulin-like growth factor (rhIGF)-I/rhIGF binding protein-3 complex improves metabolic control in subjects with severe insulin resistance. J Clin Endocrinol Metab. 2010;95(5):2113-22.

20. McDonald A, Williams RM, Regan FM, et al. IGF-I treatment of insulin resistance. Eur J Endocrinol. 2007;157 Suppl 1:S51-6.

21. Rabson SM, Mendenhall EN. Familial hypertrophy of pineal body, hyperplasia of adrenal cortex and diabetes mellitus; report of 3 cases. Am J Clin Pathol. 1956;26(3):283-90.

22. Longo N, Wang Y, Pasquali M. Progressive decline in insulin levels in Rabson-Mendenhall syndrome. J Clin Endocrinol Metab. 1999;84(8):2623-9.

23. Musso C, Cochran E, Moran SA, et al. Clinical course of genetic diseases of the insulin receptor (type A and Rabson-Mendenhall syndromes): a 30-year prospective. Med (Baltimore). 2004;83(4): 209-22.

24. Cochran E, Young JR, Sebring N, et al. Efficacy of recombinant methionyl human leptin therapy for the extreme insulin resistance of the Rabson-Mendenhall syndrome. J Clin Endocrinol Metab. 2004;89(4):1548-54.

25. Kahn CR, Flier JS, Bar RS, et al. The syndromes of insulin resistance and acanthosis nigricans. Insulin-receptor disorders in man. N Engl J Med. 1976;294(14):739-45.

26. Barbieri RL, Ryan KJ. Hyperandrogenism, insulin resistance, and acanthosis nigricans syndrome: a common endocrinopathy with distinct pathophysiologic features. Am J Obstet Gynecol. 1983;147(1):90-101.

27. Flier JS, Young JB, Landsberg L. Familial insulin resistance with acanthosis nigricans, acral hypertrophy, and muscle cramps. N Engl J Med. 1980;303(17):970-3.

28. Semple RK, Sleigh A, Murgatroyd PR, et al. Postreceptor insulin resistance contributes to human dyslipidemia and hepatic steatosis. J Clin Invest. 2009;119(2):315-22.

29. Semple RK, Halberg NH, Burling K, et al. Paradoxical elevation of high-molecular weight adiponectin in acquired extreme insulin resistance due to insulin receptor antibodies. Diabetes. 2007;56(6):1712-7.

30. Hattori Y, Hirama N, Suzuki K, et al. Elevated plasma adiponectin and leptin levels in sisters with genetically defective insulin receptors. Diabetes Care. 2007;30(11):e109. 
31. Semple R, Savage DB, O'Rahilly S. Sex hormone-binding globulin and risk of type 2 diabetes. N Engl J Med. 2009;361(27):2677. author reply $2677-2678$.

32. Semple RK, Cochran EK, Soos MA, et al. Plasma adiponectin as a marker of insulin receptor dysfunction: clinical utility in severe insulin resistance. Diabetes Care. 2008;31(5):977-9.

33. Maddux BA, Chan A, De Filippis EA, et al. IGF-binding protein-1 levels are related to insulin-mediated glucose disposal and are a potential serum marker of insulin resistance. Diabetes Care. 2006;29(7):1535-7.

34. Cochran E, Musso C, Gorden P. The use of U-500 in patients with extreme insulin resistance. Diabetes Care. 2005;28(5):1240-4.

35. • Lee JH, Huynh M, Silhavy JL, et al. De novo somatic mutations in components of the PI3K-AKT3-mTOR pathway cause hemimegalencephaly. Nat Genet. 2012;44(8):941-5. Refer to reference 10 .

36. •• Riviere JB, Mirzaa GM, O'Roak BJ, et al. De novo germline and postzygotic mutations in AKT3, PIK3R2 and PIK3CA cause a spectrum of related megalencephaly syndromes. Nat Genet. 2012;44(8):934-40. Refer to reference 10.

37. George S, Rochford JJ, Wolfrum C, et al. A family with severe insulin resistance and diabetes due to a mutation in AKT2. Science. 2004;304(5675):1325-8.

38. • Dyment DA, Smith AC, Alcantara D, et al. Mutations in PIK3R1 cause SHORT syndrome. Am J Hum Genet. 2013;93(1):158-66. One of three papers identifying PI3KR1 mutations as a the cause of the multisystem disorder, SHORT syndrome.

39. •• Thauvin-Robinet C, Auclair M, Duplomb L, et al. PIK3R1 mutations cause syndromic insulin resistance with lipoatrophy. Am J Hum Genet. 2013;93(1):141-9. Refer to reference 36.

40. • Chudasama KK, Winnay J, Johansson S, et al. SHORT syndrome with partial lipodystrophy due to impaired phosphatidylinositol 3 kinase signaling. Am J Hum Genet. 2013;93(1):150-7. Refer to reference 36.

41. Antuna-Puente B, Boutet E, Vigouroux C, et al. Higher adiponectin levels in patients with Berardinelli-Seip congenital lipodystrophy due to seipin as compared with 1-acylglycerol-3-phosphate-o-acyltransferase-2 deficiency. J Clin Endocrinol Metab. 2010;95(3):1463-8.

42. Van Maldergem L, Magre J, Khallouf TE, et al. Genotype-phenotype relationships in Berardinelli-Seip congenital lipodystrophy. J Med Genet. 2002;39(10):722-33.

43. Rochford JJ. Molecular mechanisms controlling human adipose tissue development: insights from monogenic lipodystrophies. Expert Rev Mol Med. 2010;12:e24.

44. Kim CA, Delepine M, Boutet E, et al. Association of a homozygous nonsense caveolin-1 mutation with Berardinelli-Seip congenital lipodystrophy. J Clin Endocrinol Metab. 2008;93(4):1129-34.

45. Hayashi YK, Matsuda C, Ogawa M, et al. Human PTRF mutations cause secondary deficiency of caveolins resulting in muscular dystrophy with generalized lipodystrophy. J Clin Invest. 2009;119(9):2623-33.

46. Garg A. Acquired and inherited lipodystrophies. N Engl J Med. 2004;350(12):1220-34.
47. Ali AT, Hochfeld WE, Myburgh R, Pepper MS. Adipocyte and adipogenesis. Eur J Cell Biol. 2013;92(6-7):229-36.

48. Semple RK, Chatterjee VK, O'Rahilly S. PPAR gamma and human metabolic disease. J Clin Invest. 2006;116(3):581-9.

49. Kozusko K, Patel S, Savage DB. Human congenital perilipin deficiency and insulin resistance. Endocr Dev. 2013;24:150-5.

50. Rubio-Cabezas O, Puri V, Murano I, et al. Partial lipodystrophy and insulin resistant diabetes in a patient with a homozygous nonsense mutation in CIDEC. EMBO Mol Med. 2009;1(5):280-7.

51. Oral EA, Simha V, Ruiz E, et al. Leptin-replacement therapy for lipodystrophy. N Engl J Med. 2002;346(8):570-8.

52. Petersen KF, Oral EA, Dufour S, et al. Leptin reverses insulin resistance and hepatic steatosis in patients with severe lipodystrophy. J Clin Invest. 2002;109(10):1345-50.

53. Javor ED, Ghany MG, Cochran EK, et al. Leptin reverses nonalcoholic steatohepatitis in patients with severe lipodystrophy. Hepatology. 2005;41(4):753-60.

54. Yamada K, Ikegami H, Yoneda H, et al. All patients with Werner's syndrome are insulin resistant, but only those who also have impaired insulin secretion develop overt diabetes. Diabetes Care. 1999;22(12): 2094-5.

55. Diaz A, Vogiatzi MG, Sanz MM, German J. Evaluation of short stature, carbohydrate metabolism and other endocrinopathies in Bloom's syndrome. Horm Res. 2006;66(3):111-7.

56. Huang-Doran I, Bicknell LS, Finucane FM, et al. Genetic defects in human pericentrin are associated with severe insulin resistance and diabetes. Diabetes. 2011;60(3):925-35.

57. •• Weedon MN, Ellard S, Prindle MJ, et al. An in-frame deletion at the polymerase active site of POLD1 causes a multisystem disorder with lipodystrophy. Nat Genet. 2013;45(8):947-50. First description of human POLD1 mutations associated with defective DNA repair and insulin resistance.

58. Hearn T, Spalluto C, Phillips VJ, et al. Subcellular localization of ALMS1 supports involvement of centrosome and basal body dysfunction in the pathogenesis of obesity, insulin resistance, and type 2 diabetes. Diabetes. 2005;54(5):1581-7.

59. Hearn T, Renforth GL, Spalluto C, et al. Mutation of ALMS1, a large gene with a tandem repeat encoding 47 amino acids, causes Alstrom syndrome. Nat Genet. 2002;31(1):79-83.

60. Froguel P, Zouali H, Vionnet N, et al. Familial hyperglycemia due to mutations in glucokinase. Definition of a subtype of diabetes mellitus. N Engl J Med. 1993;328(10):697-702.

61. Glaser B, Kesavan P, Heyman M, et al. Familial hyperinsulinism caused by an activating glucokinase mutation. $\mathrm{N}$ Engl J Med. 1998;338(4):226-30.

62. Senniappan S, Shanti B, James C, Hussain K. Hyperinsulinaemic hypoglycaemia: genetic mechanisms, diagnosis and management. J Inherit Metab Dis. 2012;35(4):589-601.

63. Bowles DW, Jimeno A. New phosphatidylinositol 3-kinase inhibitors for cancer. Expert Opin Investig Drugs. 2011;20(4):507-18. 